\title{
Plasma fetuin-A triggers inflammatory changes in macrophages and adipocytes by acting as an adaptor protein between NEFA and TLR-4
}

\author{
Satinath Mukhopadhyay $^{1}$ - Samir Bhattacharya ${ }^{2}$
}

Received: 18 December 2015 / Accepted: 21 December 2015 / Published online: 18 January 2016

(C) Springer-Verlag Berlin Heidelberg 2016

Keywords Fetuin-A · NEFA · TLR4

\section{Abbreviation \\ TLR4 Toll-like receptor 4}

To the Editor: We read with interest the research letter entitled 'Plasma fetuin-A does not correlate with monocyte TLR4 in humans' by Jialal and colleagues [1]. The authors state that: 'Studies using animal models, largely the work of Pal et al [2], have elegantly demonstrated that fetuin-A is the endogenous ligand for Toll-like receptor 4 (TLR4) via which lipids such as fatty acids induce insulin resistance, but there are no data in support of this in humans'. This is not, in fact, the case: in the paper referred to [2], our group provided data on human adipocytes from both diabetic and non-diabetic participants showing that fetuin-A acts as an endogenous ligand of TLR4 to induce insulin resistance. In another recent review, Stefan and Häring observed a significant correlation between circulating fetuin $\mathrm{A}$ and insulin resistance in humans [3].

Satinath Mukhopadhyay

Satinath.mukhopadhyay@gmail.com

1 Department of Endocrinology and Metabolism, Institute of Postgraduate Medical Education \& Research, Calcutta 700020, India

2 School of Life Sciences, Visva-Bharat University, Santiniketan, West Bengal, India
Jialal et al also state: 'Based on our findings, we propose that until larger studies report significant correlations between TLR4 expression in monocytes/adipose tissue and circulating fetuin-A in humans with obesity, the metabolic syndrome or diabetes, the relationship between these two proteins demonstrated in animals might not translate to humans'. In our Nature Medicine paper [2], we have clearly shown a significant correlation between plasma fetuin-A and TLR4 expression in adipocytes from abdominal subcutaneous adipose tissue of obese human subjects with type 2 diabetes.

We have shown that circulating NEFA stimulates the production of fetuin-A by the liver via NF- $\kappa$ B. Fetuin-A then forms a dimer with NEFA by acting as its binding protein. The NEFA-fetuin-A dimer finally binds to TLR-4 present on the surface of adipocytes and macrophages, resulting in the formation of a ternary complex that triggers a local inflammatory response in the adipose tissue. Upon stimulation by circulating fetuin-A, white adipose tissue attracts circulating M2 macrophages that undergo proinflammatory polarisation to M1 under the influence of fetuin-A generated locally by the inflamed adipocytes $[4,5]$. Jialal et al drew conclusions based on their studies on circulating monocytes, which are not the most appropriate model to study the NEFA-fetuin-A-TLR4 relationship [1]. Such interactions have only been reported to take place in the adipose tissue, not only by our group but also by others. In a recent paper, Trepanowski et al proposed a model wherein they suggest that: 'Fetuin-A originating from hepatocytes and adipocytes sends chemoattractant signals that induce macrophage infiltration into adipose tissue and subsequent conversion to a classically activated M1 subtype. Fetuin-A then presents fatty acids to the TLR4 receptors on both M1 macrophages and adipocytes, thereby propagating the release of proinflammatory cytokines' [6]. Moreover, our hypothesis concerned TLR4 activation and NOT expression, 
which Jialal et al went on to study [1]. Finally, their concluding remarks on fetuin-A and TLR4 expression in monocytes/ adipose tissue goes against the existing literature on the subject and unnecessarily creates a lot of confusion.

Funding There was no specific funding needed for preparing this correspondence.

Duality of interest The authors declare that there is no duality of interest associated with this manuscript.

Contribution statement Both the authors contributed significantly to the preparation of this paper and approved the final version. SM is the guarantor of this work.

\section{References}

1. Jialal I, Devaraj S, Adams-Huet B (2016) Plasma fetuin-A does not correlate with monocyte TLR4 in humans. Diabetologia 59:222-223

2. Pal D, Dasgupta S, Kundu R et al (2012) Fetuin-A acts as an endogenous ligand of TLR4 to promote lipid-induced insulin resistance. Nat Med 18:1279-1285

3. Stefan N, Häring H-U (2013) The role of hepatokines in metabolism. Nat Rev Endocrinol 9:144-152

4. Chatterjee P, Seal S, Mukherjee S et al (2013) Adipocyte fetuin A contributes to macrophage migration in adipose tissue and polarization of macrophages. J Biol Chem 288:28324-28330

5. Mukhopadhyay S, Mondal S, Kumar M, Dutta D (2014) Proinflammatory and antiinflammatory attributes of fetuin-A: a novel hepatokine modulating cardiovascular and glycemic outcomes in metabolic syndrome. Endocr Pract 20:1345-1351

6. Trepanowski JF, Mey J, Varady KA (2015) Fetuin-A: a novel link between obesity and related complications. Int J Obes 39:734-741 